\title{
ENERGY EFFICIENT SPEED AND POSITION CONTROL OF ELECTRIC DRIVES WITH PMSM
}

\begin{abstract}
A new speed and position controller respecting principles of near-energy optimal control for the drives with permanent magnet synchronous motor are developed as a contribution to the energy saving and environmental protection. Two various approaches to the energy saving controller design are analysed. The first approach is strictly based on energy optimal control theory and derives analytical solutions of the control problem. The second approach develops approximated solution for the drive position controller when the optimal speed trajectory is modified to correspond to the triangular and trapezoidal profile. This approach enables not only to compare energy demands of the individual control system design but also to exploit near-energy optimal controller for any controlled industrial drive.
\end{abstract}

Keywords: Energy optimal control, electric drives with PMSM, speed and position controller, pre-compensator.

\section{Introduction}

The consumption of electric motor represents nearly half (46\%) of global electricity consumption. Therefore, even small decrease of electric drives energy demands brought by efficient energy management, can result in significant energy savings with subsequent contribution to the protection of environment.

The main contribution of this paper is mathematical analysis of energy-optimal speed and position control of the drives with a permanent magnet synchronous motor (PMSM), taking into account stator copper losses and its possible extension towards controlled drives currently used in industry.

To complete these tasks the efficient exploitation of the time available for prescribed manoeuvre is suggested for speed controlled drives. For efficient position control a replacement of the parabolic speed profile required by analytical solution of energy optimal position control, with precisely defined trapezoidal one is proposed. Such approach enables to exploit currently used control techniques (constant torque control and constant speed control) and to extend principles of near-energy optimal control to any controlled drive with PMSM.

To get a practicable controller the influence of Coulomb friction and constant load torque on the stator current torque component is analyzed in two steps. Beside friction losses also copper losses of PMSM, which create more than $80 \%$ of total machine losses, are taking into account while the stator iron losses are neglected. As a result of analysis the useful algorithms suitable for numerical implementation, which also respect prescribed time for rest to rest manoeuvre, $\mathrm{T}_{\mathrm{m}}$ are developed.

The verification of the efficient energy management of speed and position controlled electric drives is based on principles of forced dynamics control (FDC). FDC strategy exploits principles of feedback linearization [1]. Its capability to precisely follow preplanned speed and position manoeuvre and compensate influence of load torque, which is taken into account during the planning of velocity profile, has been already experimentally verified. The proposed control structure comprises a generator of energy saving acceleration, speed and position profile, a zero dynamic lag pre-compensator and FDC position control loop. Designed nearenergy optimal position control algorithm based on symmetrical trapezoidal speed profile decreases energy demand and respects prescribed time $\mathrm{T}_{\mathrm{m}}$ for position manoeuvre.

Some significant progress on energy efficient speed control of electric drives has already been made. Model reference linear adaptive speed control of the drive with dc motor is exploited in [2] to drive output speed at any load to the maximum efficiency by controlling motor field current. A new energy saving control strategy for variable speed controlled parallel pumps based on sensorless flow rate estimation and pump operation analysis is described in [3].

Sheta et al developed minimum energy motion control systems respecting principles of optimal control theory for the drive with dc motor [4]. Similar approach was applied by Dodds et al, for the drives with PMSM [5]. Both control systems achieve

\footnotetext{
* ${ }^{1}$ Jan Vittek, ${ }^{2}$ Branislav Ftorek

${ }^{1}$ Department of Power Electrical Systems, Faculty of Electrical Engineering, University of Zilina, Slovakia

${ }^{2}$ Department of Applied Mathematics, Faculty of Mechanical Engineering, University of Zilina, Slovakia

E-mail: jan.vittek@fel.uniza.sk
} 
minimization of input energy by decreasing the losses via exploitation of the drive's kinetic energy during motion decelerating phase with respect to the speed profile and the field current. Drawbacks of these strategies are assumptions of constant friction components dependent on environmental conditions.

Due to the fact that absolute optimization of energy loss during position control isn't the main goal of this paper, the symmetrical trapezoidal speed profile is chosen and modified to achieve lower energy expenditure for a specified manoeuvre time taking into account load torque as a function of the angular velocity.

\section{Theoretical Background}

Following differential equations describe dynamics of the rotor position, $\theta_{r}$, speed $\omega_{r}$, and motor torque, $\gamma_{e}$ of the drive with PMSM:

$$
\begin{aligned}
& \frac{d \theta_{r}}{d t}=\omega_{r}, \\
& J_{r} \frac{d \omega_{r}}{d t}=\gamma_{e}-\gamma_{L}, \\
& \frac{d \gamma_{e}}{d t}=\frac{-1}{T_{S}} \gamma_{e}-\frac{k_{T}^{2}}{L_{S}} \omega_{r}+\frac{k_{T}}{L_{S}} u_{q},
\end{aligned}
$$

where $\mathrm{J}_{\mathrm{r}}$ is reduced moment of inertia to the shaft of PMSM, $\mathrm{L}_{\mathrm{s}}$ and $R_{s}$ are stator phase inductance and resistance respectively and stator time constant, $\mathrm{T}_{\mathrm{s}}$ is defined as $\mathrm{T}_{\mathrm{s}}=\mathrm{L}_{\mathrm{s}} / \mathrm{R}_{\mathrm{s}}$.

For vector controlled PMSM constant linkage magnetic flux is achieved via condition, $i_{d \text { dem }}=0$, where $i_{d \text { dem }}$ is magnetic flux component of the stator current [6]. In this case electrical torque of the motor can be expressed as:

$$
\gamma_{e}=k_{T} i_{q}
$$

where $\mathrm{k}_{\mathrm{T}}=\mathrm{c} \Psi_{P M}$ and $\mathrm{c}=3 \mathrm{p} / 2$ where $\mathrm{p}$ is number of pol-pairs, $\Psi_{P M}$ is linkage flux of permanent magnet and $\mathrm{i}_{\mathrm{q}}(\mathrm{t})$ is torque component of the stator current.

Exploiting state-space notation $\dot{x}=A x+B u$ the drive with PMSM can be described as:

$$
\left[\begin{array}{c}
\dot{\theta}_{r} \\
\dot{\omega}_{r} \\
\dot{\gamma}_{e} \\
\dot{\gamma}_{L}
\end{array}\right]=\left[\begin{array}{cccc}
0 & 1 & 0 & 0 \\
0 & 0 & \frac{1}{J_{r}} & \frac{-1}{J_{r}} \\
0 & \frac{k_{T}^{2}}{L_{s}} & \frac{-1}{T_{s}} & 0 \\
0 & 0 & 0 & 0
\end{array}\right]\left[\begin{array}{c}
\theta_{r} \\
\omega_{r} \\
\gamma_{e} \\
\gamma_{L}
\end{array}\right]+\left[\begin{array}{c}
0 \\
0 \\
\frac{k_{T}}{L_{S}} \\
0
\end{array}\right] u_{q},
$$

where the system described by (1) - (3) is completed with differential equation for constant load torque, $\dot{\gamma}_{L}=0$.

The function of optimal energy controller is to transfer control system from its initial state to the demanded state (demanded speed or position) in such a way that energy performance index I is minimized [7 and 8]. This is achieved via energy optimal control algorithm, which determines optimal excitation of the system $\mathrm{u}^{*}(\mathbf{x}, \mathrm{t})$, in this case control variable, $\mathrm{u}_{\mathrm{q}}^{*}(\mathbf{x}, \mathrm{t})$. Solution of the problem is obtained in two steps. The first step is formulation of the cost function, $\mathrm{I}_{0}$, which describes minimization problem. The developed cost function is then completed with differential equations based on state space description of the control system. If the state-space description of the system has a form:

$$
\dot{x}(t)=f[x(t), u(t)]
$$

then function $\Phi$ defined as:

$$
\phi(x, \dot{x}, u, t)=f(x, u, t)-\dot{x}=0,
$$

becomes the second part of the cost function after multiplication with Euler-Lagrange multipliers. The complete cost function I has a form:

$$
I=L_{0}(x, u, t)+\lambda^{T} \phi(x, \dot{x}, u, t),
$$

where $\mathrm{L}_{0}$ is a function describing the minimization problem and $\lambda$ represents Lagrange multipliers. Then:

$$
I_{0}=\int_{t_{0}}^{t_{1}} L_{0}(x, u, t) d t,
$$

The solution of the problem requires calculation of EulerLagrange equations, which can be found via partial derivatives of the designed cost function I for individual variables of the control system state-space description [9] as follows:

$$
\frac{\delta I}{\delta x}=\frac{d}{d t}\left(\frac{\delta I}{\delta \dot{x}}\right)
$$

Analytical approach to this problem is explained further for energy minimized speed and position control of the drive with PMSM.

\section{1. Energy Optimal Speed Control}

Energy consumption of the control system depends on minimization of copper losses given as:

$$
I_{0}=R_{s} \int i_{q}^{2} d t
$$

If relations for PMSM torque $\gamma_{\mathrm{e}}$ is used, then minimization of copper losses for prescribed manoeuvre time $\mathrm{T}_{\mathrm{m}}$ described as a function of the state-variable has a form:

$$
I_{0}=\frac{R_{s}}{k_{T}^{2}} \int_{0}^{T_{m}} \gamma_{e}^{2} d t .
$$

The complete cost function $\mathrm{I}_{\omega}$ for stator losses minimization consists of (12), in which the state space description of rotor 
speed and load torque multiplied by Lagrange multipliers is added:

$$
I_{\omega}=\frac{R_{s}}{k_{T}^{2}} \gamma_{e}^{2}+\lambda_{1}\left(J_{r} \dot{\omega}_{r}-\gamma_{e}+\gamma_{L}\right)+\lambda_{2} \dot{\gamma}_{L}
$$

and corresponding equations for individual Euler-Lagrange multipliers can be expressed as follows:

$$
\begin{aligned}
& \frac{\delta I_{\omega}}{\delta \gamma_{e}}=\frac{d}{d t}\left(\frac{\delta I_{\omega}}{\delta \dot{\gamma}_{e}}\right), \frac{2 R_{s}}{k_{T}^{2}} \gamma_{e}-\lambda_{1}=0, \\
& \frac{\delta I_{\omega}}{\delta \gamma_{L}}=\frac{d}{d t}\left(\frac{\delta I_{\omega}}{\delta \dot{\gamma}_{L}}\right), \lambda_{1}=\dot{\lambda}_{2}, \\
& \frac{\delta I_{\omega}}{\delta \gamma_{r}}=\frac{d}{d t}\left(\frac{\delta I_{\omega}}{\delta \dot{\omega}_{r}}\right), J_{r} \dot{\lambda}_{1}=0 .
\end{aligned}
$$

Using (14b) Lagrange multiplier $1_{1}$ is expressed as:

$$
\lambda_{1}=\frac{2 R_{s}}{k_{T}^{2}} \gamma_{e}
$$

From (16b) for the same Lagrange multiplier $1_{1}$ is valid:

$$
\lambda_{1}=c_{1}
$$

Exploiting (15b) and (18) multiplier $1_{2}$ is defined as:

$$
\lambda_{2}=c_{1} t+c_{2}
$$

Electrical motor torque can be expressed using (17) and (18):

$$
\gamma_{e}=\frac{k_{T}^{2}}{2 R_{s}} c_{1}
$$

Derivative of rotor speed is expressed using (2) and (20):

$$
\dot{\omega}_{r}=\frac{1}{J_{r}}\left(\frac{k_{T}^{2}}{2 R_{s}} c_{1}-\gamma_{L}\right) \text {. }
$$

If (21) is integrated, then:

$$
\omega_{r}=\frac{1}{J_{r}}\left(\frac{k_{T}^{2}}{2 R_{s}} c_{1}-\gamma_{L}\right) t+c_{3} \text {. }
$$

Constants $\mathrm{c}_{1}$ and $\mathrm{c}_{3}$ are calculated using boundary conditions as follows:

for $\mathrm{t}=0 \quad \omega_{r}=\omega_{0}$ from which $\mathrm{c}_{3}=\omega_{0}$;

for $\mathrm{t}=\mathrm{T}_{\mathrm{m}} \omega_{r}=\omega_{r \text { dem }}$ and $\mathrm{c}_{1}$ is done as:

$c_{1}=\frac{2 R_{s}}{k_{T}^{2}}\left(J_{r} \frac{\omega_{r d e m}-\omega_{0}}{T_{m}}+\gamma_{L}\right)$.

Using $c_{1}$ in relation (20) for electrical torque results in:

$$
\gamma_{e}=J_{r} \frac{\omega_{r d e m}-\omega_{0}}{T_{m}}+\gamma_{L}
$$

Subsequently, the cost performance index is defined as:

$$
I=\frac{R_{s}}{k_{T}^{2}} \int_{0}^{T m} \gamma_{e}^{2} d t
$$

and for energy optimal speed control results in:

$$
I=\frac{R_{s}}{k_{T}^{2}} T_{m}\left(J_{r} \varepsilon_{r}+\gamma_{L}\right)^{2},
$$

where $\varepsilon_{r}=\left(\omega_{r \text { dem }}-\omega_{0}\right) / \mathrm{T}_{\mathrm{m}}$ is prescribed drive acceleration.

The conclusion for this paragraph of energy optimal speed controller can be formed as: The cost performance index is direct proportional to the prescribed angular acceleration of the drive and, therefore, the longer is prescribed manoeuvre time $T_{m}$ for given change of the drive's velocity, the lower energy expenditures are required.

Results for energy optimal control of the drive speed are shown in Fig. 1. For manoeuvre time $\mathrm{T}_{\mathrm{m}}=0,25 \mathrm{~s}$ and demanded speed, $\omega_{r \text { dem }}=100 \mathrm{rads}^{-1}$ the individual time functions show: a) ideal drive acceleration and $b$ ) rotor angular speed.

\subsection{Energy Optimal Position Control}

In the case of drive's position control the complete cost function $\mathrm{I}_{\mathrm{p}}$ for stator losses minimization consists of minimization problem description (12) completed with the state space description (function $\Phi(\mathbf{x}, \dot{\mathrm{x}}, \mathrm{u}, \mathrm{t})$ ) for the rotor position and speed and load torque has a form:

$I_{p}=\frac{R_{s}}{k_{T}^{2}} \gamma_{e}^{2}+\lambda\left(J_{r} \dot{\omega}_{r}-\gamma_{e}+\gamma_{L}\right)+\lambda_{2}\left(\dot{\theta}_{r}-\omega_{r}\right)+\lambda_{3} \dot{\gamma}_{L}$

Euler-Lagrange equations for the position control are:

$\frac{\delta I_{p}}{\delta \gamma_{e}}=\frac{d}{d t}\left(\frac{\delta I_{p}}{\delta \dot{\gamma}_{e}}\right), \frac{2 R_{s}}{k_{T}^{2}} \gamma_{e}-\lambda_{1}=0$,

$\frac{\delta I_{P}}{\delta \gamma_{L}}=\frac{d}{d t}\left(\frac{\delta I_{P}}{\delta \dot{\gamma}_{L}}\right), \lambda_{1}=\dot{\lambda}_{3}$

$\frac{\delta I_{P}}{\delta \gamma_{r}}=\frac{d}{d t}\left(\frac{\delta I_{P}}{\delta \dot{\omega}_{r}}\right),-\lambda_{2}=J_{r} \dot{\lambda}_{1}$

$\frac{\delta I_{P}}{\delta \theta_{r}}=\frac{d}{d t}\left(\frac{\delta I_{P}}{\delta \dot{\theta}_{r}}\right), \dot{\lambda}_{2}=0$.

From (31b) is clear that multiplier $\lambda_{2}$ is constant:

$\lambda_{2}=c_{1}$.

Combining (30b) and (32) together for derivative of multiplier $1_{1}$ the following is valid:

$\dot{\lambda}_{1}=\frac{-c_{1}}{J_{r}}$. 
Then multiplier $\lambda_{1}$ can be expressed as:

$$
\lambda_{1}=\frac{-c_{1}}{J_{r}} t+c_{2}
$$

And, finally from (29b), Lagrange multiplier $\lambda_{3}$ is:

$$
\lambda_{3}=\frac{-c_{1}}{J_{r}} \frac{t^{2}}{2}+c_{2} t+c_{3}
$$

If (28b) and (34) are combined together, then for electrical torque the following is valid:

$$
\gamma_{e}=\frac{k_{T}^{2}}{2 R_{s}}\left(c_{2}-\frac{c_{1}}{J_{r}} t\right)
$$

The constants $\mathrm{c}_{1}$ and $\mathrm{c}_{2}$ are again calculated using boundary conditions for motor torque, which can be expressed for constant torque as:

for $\mathrm{t}=0 \quad \gamma_{e}=\gamma_{0}+\gamma_{L}$ and or $\mathrm{t}=\mathrm{T}_{\mathrm{m}} \gamma_{e}=-\gamma_{0}+\gamma_{L}$,

which results in $\mathrm{c}_{2}=\left(2 \mathrm{R}_{\mathrm{s}} / \mathrm{k}_{\mathrm{T}}^{2}\right)\left(\gamma_{0}+\gamma_{\mathrm{L}}\right)$ and $\mathrm{c}_{1}=4 \mathrm{R}_{\mathrm{s}} \gamma_{0} \mathrm{~J}_{\mathrm{r}} /\left(\mathrm{k}_{\mathrm{T}}^{2} \mathrm{~T}_{\mathrm{m}}\right)$. The required torque of PMSM has a general form:

$$
\gamma_{e}=\gamma_{0}\left(1-\frac{2}{T_{m}} t\right)+\gamma_{L}
$$

Derivative of rotor speed is expressed using (2) and (37):

$$
J_{r} \frac{d \omega_{r}}{d t}=\gamma_{0}\left(1-\frac{2}{T_{m}} t\right)
$$

If (38) is integrated with regard to the initial condition $\omega_{r}(0)=0$ and then the rotor speed is expressed as:

$$
\omega_{r}(t)=\frac{\gamma_{0}}{J_{r}}\left(t-\frac{t^{2}}{T_{m}}\right)
$$

In a similar way, using ( 1$)$ and $\mathrm{q}_{\mathrm{r}}(0)=0$ the rotor position is calculated as:

$$
\theta_{r}(t)=\frac{\gamma_{0}}{J_{r}}\left(\frac{t^{2}}{2}-\frac{t^{3}}{3 T_{m}}\right)
$$

The value of initial motor torque $\gamma_{0}$, which completes all control parameters, is expressed via the condition that at the end of the manoeuvre time $\mathrm{t}=\mathrm{T}_{\mathrm{m}}$ the rotor position should achieve a demanded position $\theta_{r \text { dem }}$. The demanded value of initial motor torque is:

$$
\gamma_{0}=\frac{6 J_{r}}{T_{m}^{2}} \theta_{r d e m}
$$

The cost performance index defined by (25) for the rotor position control is equal:

$$
I=\frac{R_{s}}{k_{T}^{2}}\left(\frac{12 J_{r}^{2}}{T_{m}^{3}} \theta_{r d e m}^{2}+\gamma_{L}^{2} T_{m}\right) .
$$

For the position control time function of consumed energy is:

$$
E_{C}=\int_{0}^{\theta_{r d e m}} \gamma_{e} d \theta_{r}=\theta_{r d e m}\left[\gamma_{0}\left(1-\frac{2}{T_{m}} t\right)+\gamma_{L}\right] \text {. }
$$

The results for energy optimal control of the drive position are shown in Fig. 2. For manoeuvre time $T_{m}=1 \mathrm{~s}$ and demanded position, $\mathrm{q}_{\mathrm{r} \text { dem }}=10$ rad individual time functions show: a) ideal drive acceleration, b) rotor speed, c) rotor angular position, which achieves a demanded position at $\mathrm{T}_{\mathrm{m}}=1 \mathrm{~s}$.

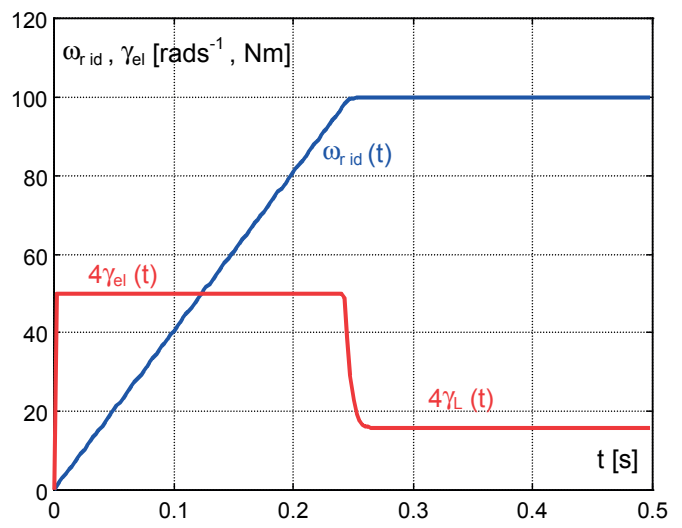

Fig. 1 Energy optimal speed control

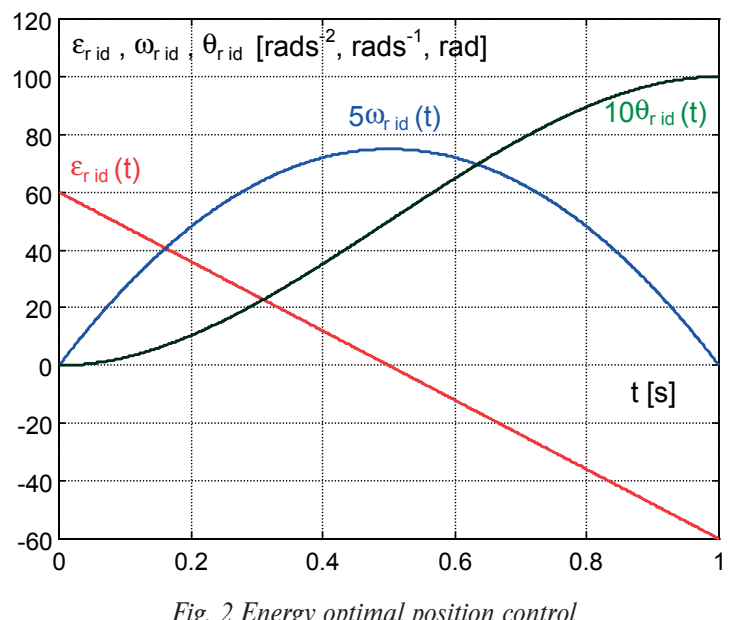

\section{Position Control System Design}

The individual approaches to energy efficient position control of electric drives differ mainly by description of velocity time profile. A controller for dynamical systems with energy optimization performances was proposed in [10].

A generation of three steps velocity function based on efficient binary search algorithm minimizing energy demands was proposed by Kim et al for a battery powered wheeled mobile 
robot [11] in order to extend battery run time. A new objective function considering practical energy consumption was developed together with an efficient iterative search algorithm finding its numerical solution. Trapezoidal velocity function with constant acceleration, deceleration and cruising speed was used by Dodds for practicable system based on sliding mode control of the PMSM drive position [12]. Both papers report nearly $30 \%$ less energy loss if compared with a conventional concept of control.

Minimization of the total dissipated energy for a position control system with reduction gear taking into account Coulomb and viscous friction was proposed by Zhu et al [13]. The optimal drive velocity and current functions are obtained as a function of the optimal zero crossing time. The comparison of the dc motor drive consumptions for optimized algorithm and trapezoidal velocity profile revealed energy savings of optimized algorithm, which were proportional to a moment of inertia.

\subsection{Speed Profile Simplification}

To avoid continues speed control during a position manoeuvre often the control on motor torque constant value with one switching in the middle of manoeuvre time is used to perform near-time optimal position control. Time functions for acceleration, speed and rotor position are shown in Fig. 3 .

A more efficient energy saving solution for a near-energy optimal position control is proposed exploiting symmetrical trapezoidal function of rotor speed as shown in Fig. 4 together with other two control variables. In this case the control tasks are completed with control on constant torque during acceleration and deceleration intervals and control on constant cruising speed in the middle interval, which are the most common control techniques used in drive control.

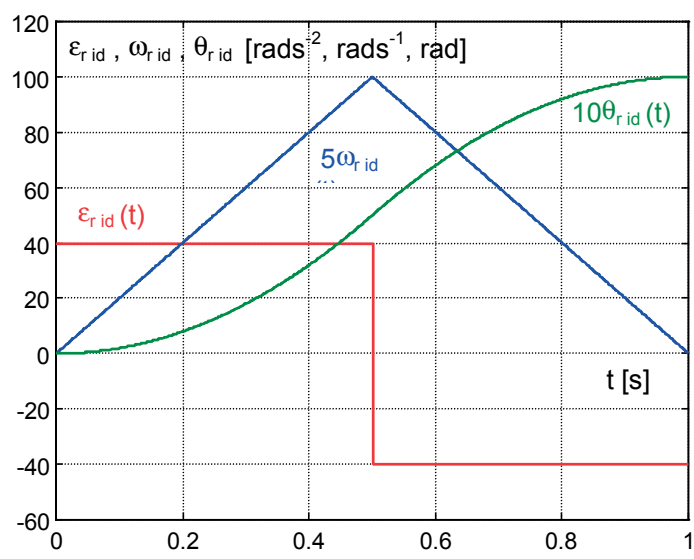

Fig. 3 Energy saving control, triangular speed profile

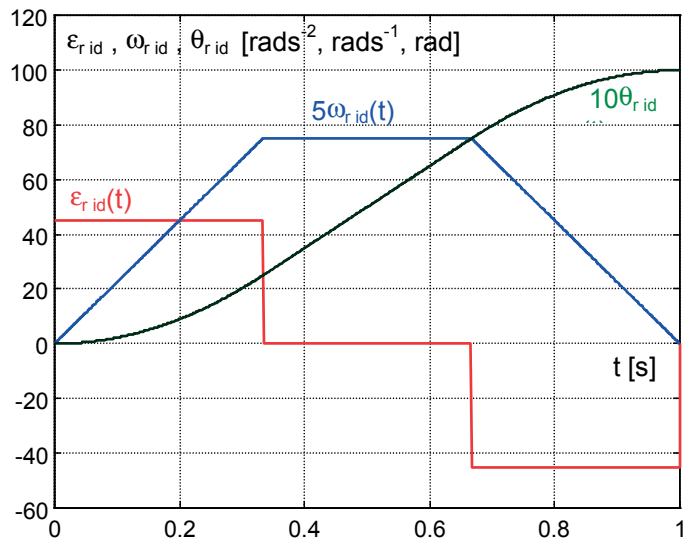

Fig. 4 Energy saving control, trapezoidal speed profile

Replacement of a continuously varying energy-optimal speed profile with symmetrical trapezoidal one was confirmed through analytical analysis of the Joule losses in stator winding and losses to cover Coulomb and viscous friction in [14]. Similar approach was applied to the drive with friction or load torque as a quadratic function of rotor speed [15]. Newton-Raphson search algorithm is exploited to find acceleration time with minimal energy expenditures.

The main goal of the position control system suitable for any described energy saving position control strategy is to track the best designed position, velocity and acceleration functions. The position control system shown in Fig. 6 contains an energy saving position generator, a zero dynamic lag pre-compensator and FDC based position control loop.

\subsection{Forced Dynamics Speed Control}

Speed control loop of the PMSM, which is designed using FDC, respects the vector control conditions [16 and 17]. Based on feedback linearization the rotor speed obeys (2) and the differential equation describing the closed loop dynamical behaviour has, therefore, linear first order dynamics (44) where $T_{\omega}$ is the prescribed time constant and $\omega_{r d e m}$, is the demanded rotor speed:

$$
\ddot{\theta}_{r}=\frac{1}{T_{\omega}}\left(\dot{\theta}_{r d}-\dot{\theta}_{r}\right) .
$$

Setting $i_{d}=0$ for vector control of the PMSM up to the nominal speed and equating the RHS of (2) and (44) yields the FDC speed control algorithm:

$$
i_{d \text { dem }}=0
$$

and

$$
i_{q d e m}=\frac{1}{c \Psi_{P M}}\left[\frac{J}{T_{\omega}}\left(\dot{\theta}_{r d}-\dot{\theta}_{r}\right)+\hat{\Gamma}_{L}\right] .
$$


Current components $i_{d}=i_{d \text { dem }}$ and $i_{q}=i_{q \text { dem }}$ are regarded as the control variables which means that the stator current equations are eliminated from the control system design. To estimate net load torque on the motor shaft the torque observer with filtering effect is used.

\subsection{Forced Dynamics Position Control}

For the design of a position control loop the FDC speed control loop is replaced by its ideal transfer function and completed with a kinematic integrator. Parameters of this loop are adjusted using Dodds's settling time formula [18]:

$$
\begin{aligned}
& \frac{\theta_{r}(s)}{\theta_{r d}^{\prime}(s)}=\left.\left[\frac{1}{1+s \frac{T_{s \theta}}{1,5(1+n)}}\right]\right|_{n=2} ^{n}= \\
& \frac{1}{\frac{4 T_{s \theta}^{2}}{81} s^{2}+\frac{4 T_{s \theta}}{9} s+1} .
\end{aligned}
$$

Converting (46) into time domain yields the second order differential equation for the rotor position:

$$
\ddot{\theta}_{r}=\frac{81}{4 T_{s \theta}^{2}}\left(\theta_{r d}^{\prime}-\theta_{r}\right)-\frac{9}{T_{s \theta}} \dot{\theta}_{r} .
$$

FDC law of the rotor angle is obtained by substituting (44) for LHS of (47) and solving it for the demanded rotor speed as a control variable $\dot{\theta}_{r d}$ :

$$
\dot{\theta}_{r d}=\left(1-\frac{9 T_{\omega}}{T_{s \theta}}\right) \dot{\theta}_{r}+\frac{81 T_{\omega}}{4 T_{s \theta}^{2}}\left(\theta_{r d}^{\prime}-\theta_{r}\right)
$$

A block diagram for FDC of rotor position is shown in Fig. 5.

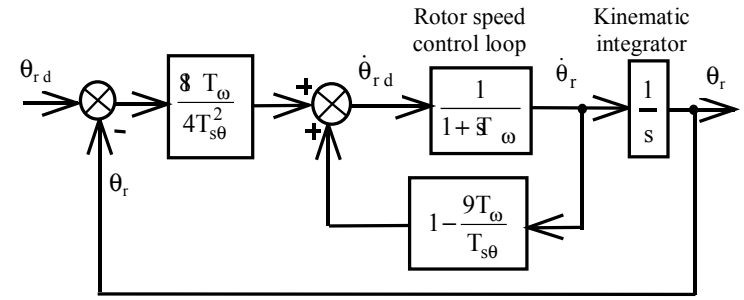

Fig. 5 Block diagram for FDC position control

\subsection{Pre-compensator and Torque Observer}

Closed loop dynamic of a position controller is prescribed by (46), therefore, the pre-compensator has then the inverse transfer function as follows:

$$
F_{P C}(s)=\frac{\theta_{r d}(s)}{\theta_{r}(s)}=s^{2} \frac{4 T_{s}^{2}}{81}+s \frac{4 T_{s}}{9}+1 .
$$

Implementation of the pre-compensator to the overall energy saving position control block diagram is shown in Fig. 6. The design of observer was published in previous work and details can be found in [19].

\section{Verification by Simulations}

Energy demands of the designed energy-optimal control system and its two possible modifications as near-energy optimal control are investigated to observe their tracking abilities as well as energy expenditures.

\subsection{Tracking Abilities Evaluation}

All the simulations presented are carried out with zero initial state variables. A step position demand $\theta_{\mathrm{rdem}}=10 \mathrm{rad}$ with prescribed manoeuvre time $\mathrm{T}_{\mathrm{m}}=0.25 \mathrm{~s}$ was applied to investigate response of the control system. The computational step of simulations is $\mathrm{h}=1 \mathrm{e}-4 \mathrm{~s}$. PMSM parameters are listed in the Appendix.

Ideal state-variables produced by an energy saving profiles generator are shown in Fig. 7, in which time functions of speed and position responses of the drive for energy optimal and another two near-energy optimal control techniques can be seen. It's clear from subplot a) that the properly adjusted trapezoidal approximation of parabolic speed increase (and decrease) of energy-optimal control are very close and absolute error, $\mathrm{e}_{\omega}(\mathrm{t})$ doesn't exceed $3 \%$. Also the magnitude of speed for both time functions is identical.

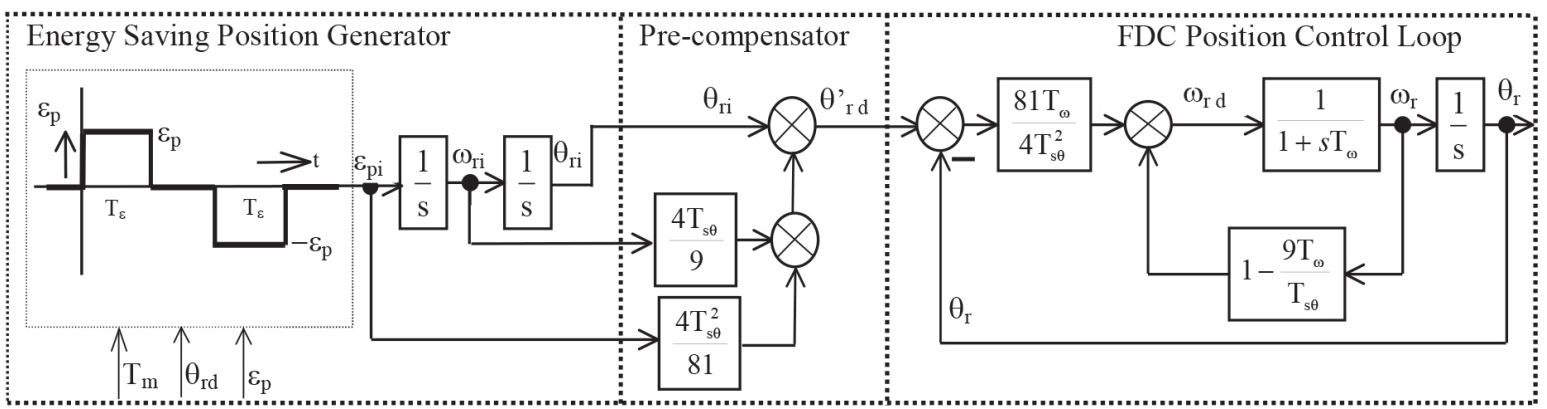

Fig. 6 Overall PMSM position control system block diagram for losses optimization 


$$
\omega_{p 1,2}=\frac{3 \theta_{r d}}{2 T_{m}}, \omega_{p 3}=\frac{2 \theta_{r d}}{T_{m}} .
$$

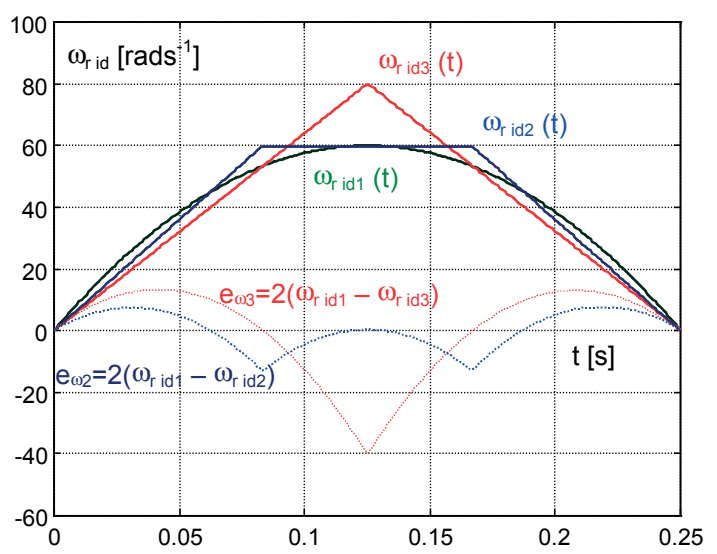

a) angular speed

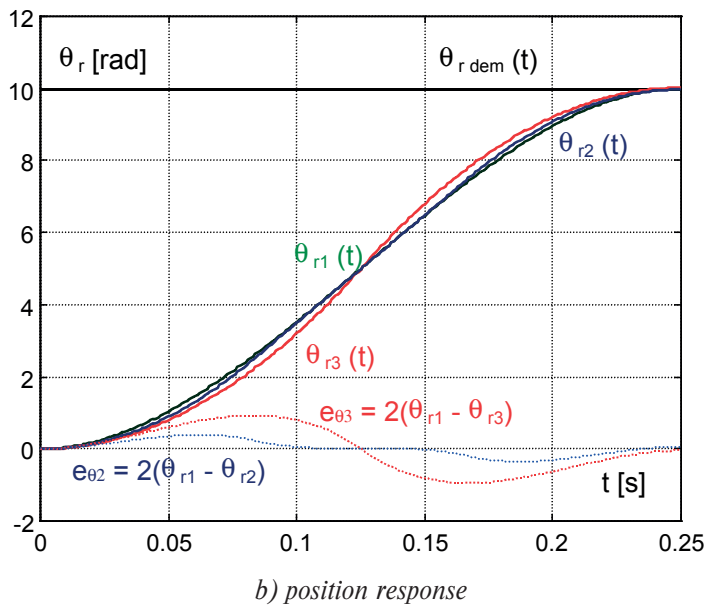

Fig. 7 Ideal state-variables for energy optimal and near-energy optimal control

As it was observed from simulations tracking abilities of the designed overall control systems are capable to follow desired state variables with negligible dynamic lag.

\subsection{Evaluation of Energy Consumption}

Described energy saving control algorithms for PMSM were tested by simulation. Total energy consumption of the drive was evaluated as:

$$
W_{t}=\frac{3}{2} \int_{0}^{T_{m}}\left(u_{q} i_{q}+u_{d} i_{d}\right) d t .
$$

To check the correctness of energy computation, all output drive's energy components including energy of chokes and energy of rotational parts were summed in a good agreement with total input energy (51). Results of energy consumption evaluation are shown for idle running drive and drive loaded with nominal torque in Fig. 8. As Fig. 8 shows the total energy consumption for idle running and nominally loaded energy optimal controlled drive is $\mathrm{W}_{\mathrm{t} 0}=188 \mathrm{Ws}$ and $\mathrm{W}_{\mathrm{t} \text { nom }}=580 \mathrm{Ws}$ respectively. These values were taken as a base for comparison of other two nearenergy optimal control techniques.

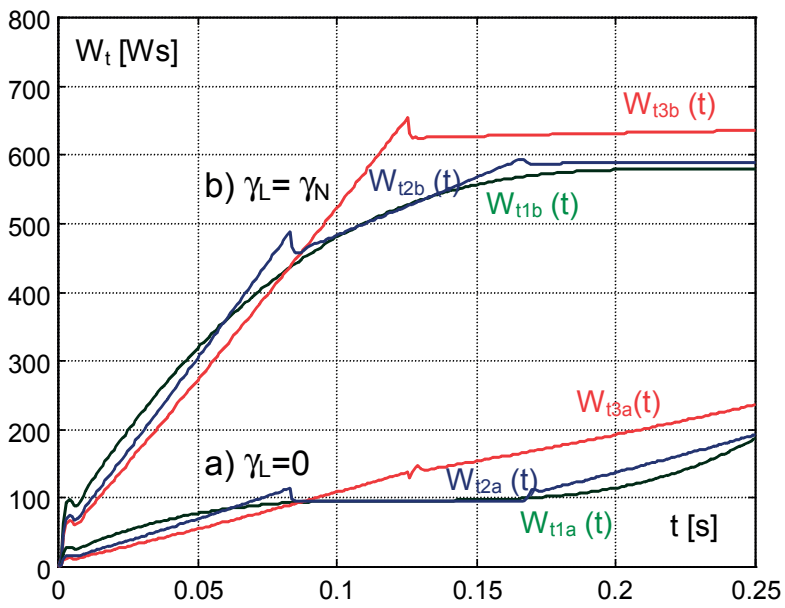

Fig. 8 Total energy consumption for prescribed position manoeuvre

Further observations show that the triangular speed profile is the most energy demanding. Its energy expenditures are by $25.8 \%$ and $12.5 \%$ higher for no-loaded and nominally loaded drive respectively.

On the contrary, the designed trapezoidal speed profile with respect to load conditions has only by $2.4 \%$ and $2.7 \%$ respectively higher energy demands if compared with the energy optimal control. It is necessary to note that energy expenditures are proportional to the moment of inertia, therefore the energy expenditures can differ for various moments of inertia.

\section{Conclusion}

A new energy saving speed and position controller respecting near-energy optimal control of PMSM were designed and verified by simulations.

Both control algorithms are based on losses minimization, therefore, their validity can be extended to any load with constant torque. Derived relations mathematically justify the choice of symmetrical trapezoidal speed profile selection for a position manoeuvre with prescribed position time.

The verification by simulations confirmed the lowest energy expenditures of the drive with energy-optimal control. Mutual 
comparison of near-energy optimal position control with triangular and trapezoidal angular speed profiles has shown higher energy savings of the symmetrical trapezoidal angular speed profile.

Moreover, a developed energy saving position control system is practicable due to its simplicity and implementation ability exploiting the most common modes for controlled drives as control on constant torque and control on constant speed.

\section{Acknowledgement}

The authors wish to thank the Slovak Grant Agency VEGA for funding the project No.1/0355/11 'Optimal Control Techniques for Decreasing Losses of A.C. Drives'.

\section{Appendix}

Parameters of PMSM are: winding resistance, $\mathrm{R}_{\mathrm{s}}=1.3 \Omega$, inductances, $\mathrm{L}_{\mathrm{d}}=14.4 \mathrm{mH}, \mathrm{L}_{\mathrm{q}}=16.3 \mathrm{mH}$, permanent magnet flux, $\Psi_{\mathrm{PM}}=0.13 \mathrm{~Wb}$, number of pole pairs, $\mathrm{p}=5$ and moment of inertia, $\mathrm{J}_{\mathrm{r}}=0.005 \mathrm{kgm}^{2}$.

\section{References}

[1] ISIDORI, A.: Nonlinear Control Systems, London: Springer-Verlag Berlin, 2001. ISBN 3-5400-19916-3 ${ }^{\text {rd }}$ edition.

[2] GAJENDRAN, F., GEORGE S.: A Simple Linear Adaptive Speed Control of Energy Efficient DC Drives. Electric Machines and Drives, 1999. Intern. Conference IEMD ‘99, Seattle, WA, ISBN 0-7803-5293-9.

[3] VIHOLAINEN, J., TAMMINEN, J., ABONEN, T., AHOLA, J. et all: Energy-efficient Control Strategy for Variable Speed-driven Pumping System. Springer, Energy Efficiency, vol. 6, 2013, pp. 495-509.

[4] SHETA, M., AGARWAL, V. A., NATARAJ, V. S. P.: A New Energy Optimal Control Scheme for a Separately Excited DC Motor Based Incremental Motion. Intern. J. of Automation and Computing, vol. 6, No. 3, August 2006, pp. 267-276, ISSN 1476-8186.

[5] DODDS, S. J.: Sliding Mode Vector Control of PMSM Drives with Minimum Energy Position Following. Proc. of European power Electronic, Power Electronics and Motion Control, EPE-PEMC Conference, Poznan, 2008, pp. 2559-2566.

[6] FILKA, R., BALAZOVIC, P., DOBRUCKY, B.: A Sensorless PM Synchronous Motor Drive for Electric Washers, Communications - Scientific Letters of the University of Zilina, 2007, No. 1, pp. 24-32.

[7] WANG, Y., UEDA, K., BORTOFF, A. S.: On the Optimal Trajectory Generation for Servomotors: A Hamiltonian Approach. Proc. of the IEEE Conference on Decision and Control, 2012, pp. 7620-7625, ISSN: 0191-2216.

[8] BIVOL, I., VASILACHE, C.: The Application of Euler - Lagrange Method of Optimization for Electromechanical Motion Control. The Annals of Dunarea de Joss University of Galati, vol. 3, pp. 5-11, ISSN 1221-454X.

[9] GULDAN, V., MARCOKOVA, M.: Orthogonal Polynomials and Related Special Functions Applied in Geosciences and Engineering Computations. Communications - Scientific Letters of the University of Zilina, No. 1, 2010, pp. 12-15.

[10] FEDOR, P., PERDUKOVA, D.: Energy Optimization of a Dynamic System Controller. Advances in Intelligent Systems and Computing, vol. 189, AISC, 2013, pp. 361-369, ISSN: 21945357.

[11] KIM, C. H., KIM, B. K.: Energy Saving 3-steps Velocity Control Algorithm for Battery-powered Wheeled Mobile Robot. Proc. of 2005 IEEE Intern. Conference on Robotics and Automation, Barcelona, Spain 2005, pp. 2375-2380.

[12] DODDS, S. J., SOORIYAKUMAR, G., PERRYMAN, R.: Sliding Mode Minimum Energy Position Controller for Permanent Magnet Synchronous Motor Drives. WSEAS Transactions on Systems and Control, No. 4, vol. 3, April 2008, pp. 299-309. ISSN 1991-8763.

[13] ZHU, Y., ZHU, X., IZUMI, T., KANESAKA, M.: Optimal Velocity Function Minimizing Dissipated Energy Considering All Friction in a Position Control System. J. of Robotics and Mechatronics, vol. 10, Jan. 2007, pp. 97-105. ISSN 0915-3942.

[14] VITTEK, J., BRIS, P.: Energy Saving Position Control Algorithms for PMSM Drives with Coulomb and Viscous Friction. Proc. of $10^{\text {th }}$ IEEE Intern. Conference on Control and Automation, Hangzhou, China, 2013. ISBN 978-1-4673-4706-8.

[15] VITTEK, J., BRIS, P., BIEL, Z., HRKEL, M.: Energy Saving Position Control Algorithms for PMSM Drives with Quadratic Friction. Proc. of IEEE Africon intern. conference on Control and Automation, Mauritius, 2013 pp. 1001-1006. ISBN 978-1-4673-5940-5

[16] LEONHARDT, W.: Control of Electrical Drives. Springer-Verlag, Berlin, 2001, ISBN 3-540-41820-2.

[17] BRANDTSTETTER, P., KRECEK, T.: Speed and Current Control of Permanent Magnet Synchronous Motor Drive using IMC Controllers. Advances in Electrical and Computer Engineering, No. 4, 2012, pp. 3-10, ISSN 1582-7445.

[18] DODDS, S. J.: Settling Time Formulae for the Design of Control Systems with Linear Closed Loop Dynamics. Proc. of the Intern. Conference AC\&T - Advances in Computing and Technology, University of East London, 2007, pp. 31-39. ISSN 1350245X.

[19] VITTEK, J., DODDS, S. J.: Forced Dynamics Control of Electric Drives, EDIS Publishing Centre of Zilina University, Slovakia, 2003, available at http://www.kves. uniza.sk/ (e-learning). 